\title{
CONDITION BASEd MAINTENANCE OF TURBINE AND COMPRESSOR OF A CODLAG NAVAl Propulsion System Using DeeP NEURAL NETWORK
}

\author{
Palash Pal ${ }^{1}$, Rituparna Datta ${ }^{2}$, Aviv Segev $^{2}$, and Alec Yasinsac ${ }^{2}$ \\ ${ }^{1}$ University Institute of Technology, Burdwan University, West Bengal, India \\ ${ }^{2}$ Department of Computer Science, University of South Alabama, 150 Jaguar \\ Drive, Mobile, AL 36688, USA
}

\begin{abstract}
System and sub-system maintenance is a significant task for every dynamic system. A plethora of approaches, both quantitative and qualitative, have been proposed to ensure the system safety and to minimize the system downtime. The rapid progress of computing technologies and different machine learning approaches makes it possible to integrate complex machine learning techniques with maintenance strategies to predict system maintenance in advance. The present work analyzes different methods of integrating an Artificial Neural Network (ANN) and ANN with Principle Component Analysis (PCA) to model and predict compressor decay state coefficient and turbine decay state coefficient of a Gas Turbine (GT) mounted on a frigate characterized by a Combined Diesel-Electric and Gas (CODLAG) propulsion plant used in naval vessels. The input parameters are GT parameters and the outputs are GT compressor and turbine decay state coefficients. Due to the presence of a large number of inputs, more hidden layers are required, and as a result a deep neural network is found appropriate. The simulation results confirm that most of the proposed models accomplish the prediction of the decay state coefficients of the gas turbine of the naval propulsion. The results show that a consistently declining hidden layers size which is proportional to the input and to the output outperforms the other neural network architectures. In addition, the results of ANN outperforms hybrid PCAANN in most cases. The ANN architecture design might be relevant to other predictive maintenance systems.
\end{abstract}

\section{KEYWORDS}

Condition based maintenance, Neural Network, Deep neural network, Principle Component Analysis(PCA), Naval propulsion

\section{INTRODUCTION}

Maintenance of any dynamic system and its components is always a very important and crucial task as failure of one or more components may damage the whole system. For a complex system like a naval vessel, maintenance demands a high level of expertise, which is sometimes difficult to achieve $[1,2]$. Due to the rapid progress of computing technologies and different machine learning approaches, today it is possible to integrate complex machine learning techniques and maintenance strategies, thus helping to identify new standards and raise the level of performance $[3,4]$. The predictive maintenance of a physical system is significant to ensure identification of

Dhinaharan Nagamalai et al. (Eds) : CCSEIT, ICBB, DMDB, AIAP, CSNA - 2019 
any fault that may occur during operation. Predictive maintenance helps to improve efficiency as well as the reliability of the system performance [5,6]. A system like the propulsion plant of a naval destroyer should always be in proper working condition without any interruption, and at the same time its performance should be monitored continuously to avoid any unexpected breakdown $[7,8]$. Predictive maintenance is a good maintenance strategy under such a situation. Sensors integrated within the system components can provide streaming data in real time without interrupting the system work and performance [9]. Received sensor data can then be analyzed to monitor the condition of different system components and to predict the risk of any potential future failure.

In this paper, we analyzed different methods to predict the maintenance strategy for a CODLAG propulsion system $[10,11]$ used in naval vessels. Diverse background knowledge is required to understand the detailed working principles of naval vessels. A basic knowledge of different types of naval vessel propulsion-systems (specifically CODLAG propulsion system in our case) and working principles of gas turbines and knowledge about system maintenance along with available maintenance strategies are necessary.

In this work we show the capability of machine learning strategies like deep learning to predict the maintenance of a propulsion plant of a naval vessel. The details of the CODLAG propulsion system are shown in Fig. 1. CODLAG is a hybrid propulsion system and uses an electric motor, powered by a diesel generator, to cruise or to run the ship silently. A gas turbine is used when high speed is required, for example during an emergency situation faced by a naval force. Electric motors, powered by the diesel generators are connected to the propeller shaft. When high speed is required, gas turbine is engaged using cross connecting gearbox to rotate the shaft. While cruising, clutches are used to disengage the drive train of the turbine. Electric motors are very effective when wide range of revolution is required. Direct connection between the electric motors and shaft can be established reducing complexity of gear box.

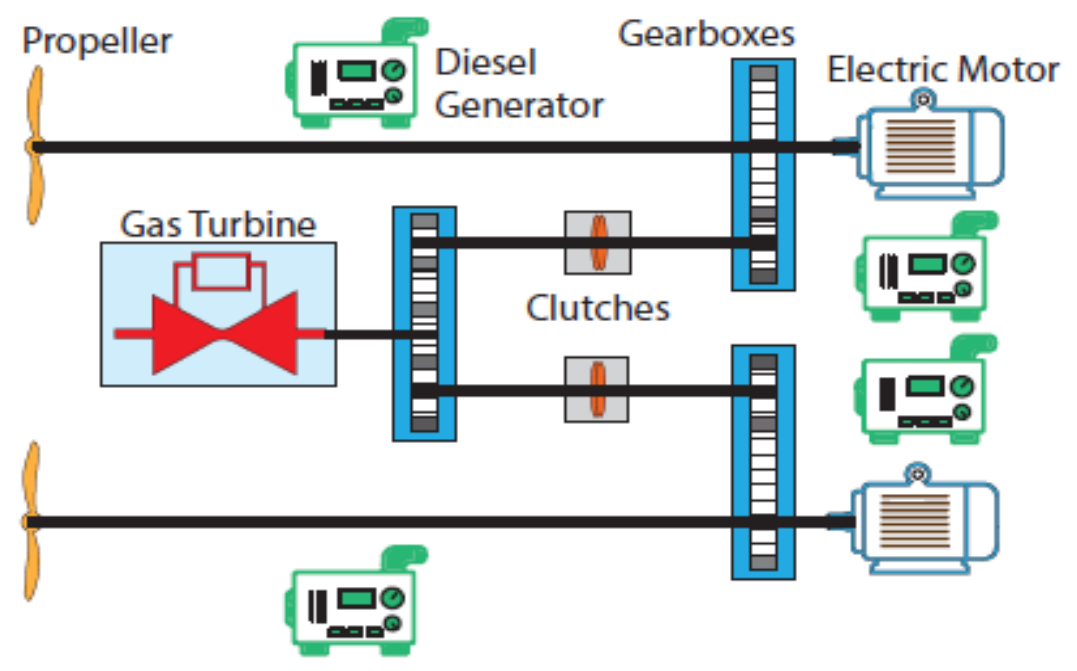

Fig. 1. Working principle of the CODLAG propulsion system.

Propulsion means forcing an object to move in a forward direction. The propulsion system produces the necessary thrust to move the object to forward [12]. The marine propulsion system generates the required thrust to move forward a ship or vessel across the water [13, 14]. There are different types of marine propulsion systems available. They are diesel propulsion [15], GT propulsion [16], nuclear propulsion [17], solar propulsion [18], steam turbine propulsion [19], 
diesel-electric propulsion [20], etc. Different issues are taken into consideration when selecting a suitable propulsion system for a vessel [21]. Some of the issues are range of ship speed, pollution emitted, noise produced, number of people needed to maintain and operate the ship, how long the ship can be away from the base, etc.

Neural networks have been used to predict maintenance in many research studies. Lucifred et al. [22] compared linear multiple regression, the dynamic kriging technique, and neural networks for predictive maintenance. The results showed that integration kriging and the ANN technique achieved best results. Javadpour and Knapp [23] also used NN for faults diagnosis in an automated manufacturing environment. The performance is tested with time series and real time machine vibration data.Wu et al. [24] proposed an integrated $\mathrm{NN}$ based decision support system of rotational equipment for maintenance prediction.

The organization of the rest of the present paper is as follows. Section 2 discusses the details of naval propulsion and maintenance. Then in Section 3, we provide the details of the data-set used in the present study. The simulation results are shown in Section 4. The future scope along with the conclusion is in Section 5.

\section{Naval Propulsion And Maintenance}

In this paper, we are interested in the CODLAG propulsion system. Naval forces in many countries use the CODLAG propulsion system in their frigate or destroyer. The Type 23 frigate used by the United Kingdom Royal Navy, and the F125 Baden-Wrttemberg-class frigate used by the German Navy, FREMM used by the Italian Navy implement CODLAG propulsion system. CODLAG is a hybrid propulsion system. CODLAG uses an electric motor, powered by a diesel generator for cruising or to run the ship silently. A gas turbine is used when high speed is required, for example during an emergency situation faced by a naval force. GT has three components: air compressor, combustion chamber, and turbine. The compressor absorbs air from the atmosphere and increases the pressure. The pressurized air then enters into the combustion chamber. Inside the combustion chamber there are fuel injectors and high intensity spark igniters. Fuel injectors inject a steady stream of fuel into the chamber. The injected fuel is mixed with air entered from the compressor. The injected fuel-air mixture is ignited by the igniters present in the combustion chamber. In this process, very hot and highly pressurized gas is produced. The produced gas is then expanded through an exhaust nozzle. The resulting hot, high velocity exhaust gas from the combustion chamber drives the turbine. The GT produces mechanical energy to rotate the shaft. This shaft having enormous torque turns the propeller or generator [12, 25]. Altosole et al. [26] simulated a numerical 3 degree of freedom ship system during transients and off design conditions.

The propulsion system of a vessel is very important as the overall behaviour of the ship depends exclusively on its propulsion system. So the proper maintenance of the propulsion system of the vessel is a crucial and unavoidable task. There are different types of maintenance strategies. Maintenance is a term that includes many activities such as system and component testing, adjustment, repair and replacement of system components, rebuilding etc. to retain the overall system performance. Maintenance strategies are divided mainly into three categories. They are preventive or scheduled maintenance, corrective maintenance, and predictive maintenance. Preventive maintenance is a routine check-up of a system and its components while it is in working condition. Preventive maintenance is performed at regular time intervals. That is why it is also called scheduled maintenance. Corrective maintenance is performed on a system once the system or its components break down. Recently, due to advances in science and technology, a third type of maintenance strategy, predictive maintenance, has been introduced. In a predictive maintenance strategy, sensors are used in a system or system component to monitor key 
parameters related to the system in real time. System data is collected continuously and analyzed using different techniques available to evaluate the system health and to predict possibilities of any future failure(s) before it happens. Condition Based Maintenance (CBM) falls within the predictive maintenance strategy [27]. CBM continuously monitors system and system components to decide when and which components need to be serviced. Based on the three different maintenance strategies discussed, a few conclusions can be drawn. Preventive maintenance is complex to process and time consuming to perform if the system is itself a complex system. Moreover, as the maintenance is performed before failure, the system and components may be in good shape when it is checked, resulting in wastage of money. Corrective maintenance is sub-optimal as it is performed only after system or system component(s) fail. In this case, already damaged system component(s) may damage other components, resulting in multiple failures. The corrective maintenance strategy is also not a good fit for a system like the propulsion plant of a frigate, which cannot afford sudden failure, as it must always be in good working condition to counter any emergency situation that may arise. Predictive maintenance or condition based maintenance is the best choice for our naval CODLAG propulsion system.

\section{Naval Propulsion Data-Set}

For the experiments conducted in this paper, we collected data from the UCI Machine Learning repository [28]. Data has been generated from a sophisticated simulator of a GT, mounted on a frigate characterized by a CODLAG propulsion plant [29]. The different blocks forming the complete simulator (Propeller, Hull, GT, Gear Box, and Controller) have been developed and fine-tuned over the years on several similar real propulsion plants. In view of these observations, the available data is in agreement with a possible real vessel. A series of measures (16 featured vectors) which represents the state of the system has been analyzed in this experiment using an Artificial Neural Network to predict compressor decay state coefficient and turbine decay state coefficient.

The 16-featured input vector contains the following parameters which effect the performance of the gas turbine and its compressor and could lead to decay:

1. Lever position

2. Ship speed [knots]

3. CGT shaft torque [k N m]

4. GT rate of revolutions [rpm]

5. Gas generator rate of revolutions [rpm]

6. Starboard propeller torque [ $\mathrm{k} \mathrm{N} \mathrm{m}]$

7. Port propeller torque [k N m]

8. High pressure (HP) turbine exit temperature [C]

9. GT compressor inlet air temperature [C]

10. GT compressor outlet air temperature [C]

11. HP turbine exit pressure [bar]

12. GT compressor inlet air pressure [bar]

13. GT compressor outlet air pressure [bar]

14. GT exhaust gas pressure [bar]

15. Turbine injection control [\%]

16. Fuel flow $[\mathrm{kg} / \mathrm{s}]$

The goal is to extend the life expectancy of the gas turbine and the gas turbine compressor by optimizing decay state coefficients, which are:

1. GT compressor decay state coefficient

2. GT turbine decay state coefficient 
The kernel density plots for both outputs are shown in Fig. 2 and Fig. 3. The kernel density plot represents the probability density distribution of given data. The total area under the curve is one. The two plots here represent the probability density distribution of our two output variables. To find the probability of a value falling within an interval, we need to calculate the area under the curve within that interval. From the kernel density plots, it is clear that both of our outputs are uniformly distributed.

A scatter plot matrix of all input and output variables is shown in Fig. 4. The scatter plot matrix here is a visual representation of the correlation between pairs of columns from a dataset. It shows a pairwise scatter plot for each pair of columns in our dataset. From the scatter plot diagram here, We show below that all of our input features are strongly correlated with each other.

All the small boxes represent the scatter plot with two different features. There are 16 inputs and 2 outputs, labelled as V1, V2, ..., V18. Columns V9 representing the Gas Turbine compressor inlet air temperature (C) and column V12 representing the Gas Turbine compressor inlet air pressure (bar) have constant values for all data items. Column V6 and column V7 represent starboard propeller torque and port propeller torque respectively. As starboard propeller torque and corresponding port propeller torque are the same for all data values, V6 and V7 are found to be identical. The data is plotted using a scatter

\section{Compressor Decay State Coefficient}

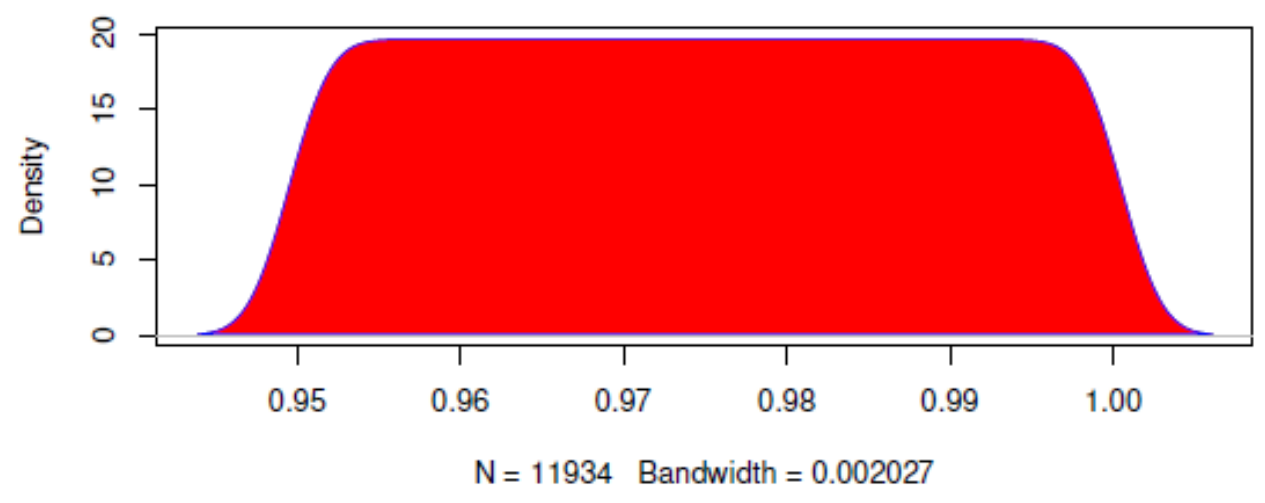

Fig. 2. Kernel density plot for compressor decay state coefficient.

plot matrix, showing a visual explanation of the relationship between pairs of variables. The figure shows pairwise scatter plot for each pair of the columns in the dataset. The scatter plot matrix of size 18X18 contains all the scatter plots between all the possible pairs of variables. From the scatter plot, we can analyze the correlation between the different variables. If two variables are not correlated, then the scatter plot between them will contain data points scattered at random without forming any pattern. But for two variables which are correlated, the scatter plot will contain data points forming some pattern. If the pattern falls on a line, then the two variables are linearly correlated, else they are nonlinearly correlated. In the diagram, we can see most of the variables are almost linearly correlated. As all the data items in column V9 and V16 are constant, all the scatter plots containing V9 and V16 form a linear pattern, parallel to the axis. As column V6 and V7 are identical, they form identical scatter plots. V17 and V18 are output columns and outputs are not correlated with any single input. As a result, scatter plots of the output variables cover the whole box, without forming any pattern. Numbers and lines coming 
out of scatter plots on top, bottom, left and right represent the scale of data items, which due to lack of space appear in only some of the plots.

\section{Simulation Results}

The range of decay of the compressor and turbine were sampled with a uniform grid of precision (0.001) to have good granularity of representation. In particular, for the compressor decay state discretization, the coefficient was investigated in the domain [0.95:1] and for the turbine, decay state coefficient is in the domain [0.975:1]. We used a multilayer feed forward neural network to predict both of our outputs simultaneously against our 16-featured input. While running our code using the default neural network configuration

Gas Turbine Decay State Coefficient

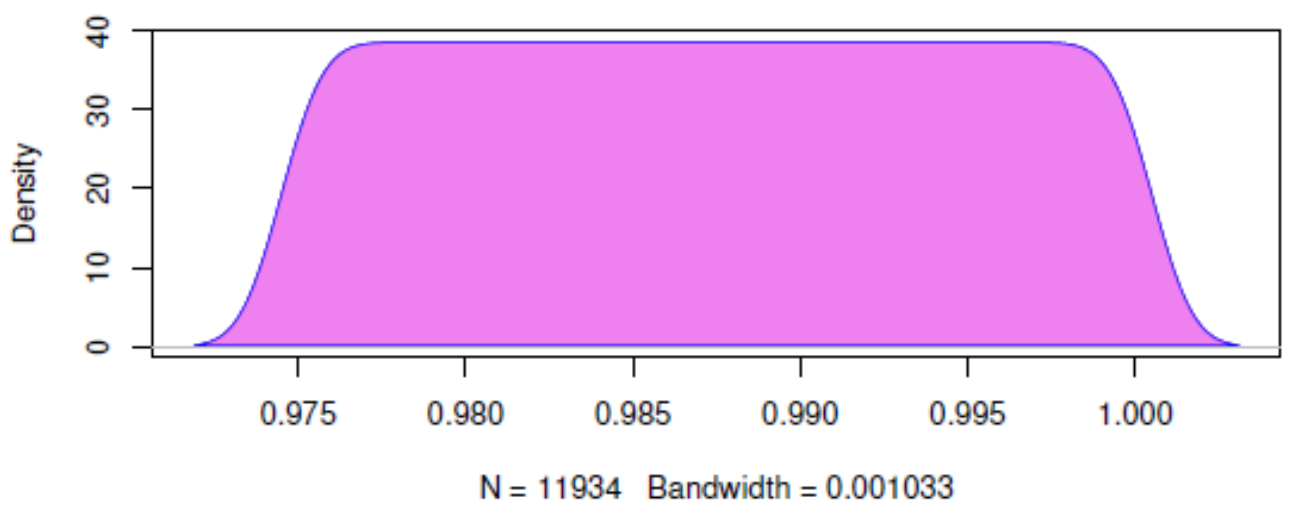

Fig. 3. Kernel density plot for turbine decay state coefficient.

\begin{tabular}{|c|c|c|c|}
\hline Architecture & MSE1 & MSE2 & Average MSE \\
\hline $8-6-4-2$ & 0.00017 & 0.00013 & 0.00015 \\
\hline $8-6-4$ & 0.00011 & 0.00017 & 0.00014 \\
\hline $8-6$ & 0.00012 & 0.00020 & 0.00016 \\
\hline $6-4$ & \multicolumn{3}{|c|}{ Convergence failed } \\
\hline
\end{tabular}

Table 1. Comparison between different neural network architectures in term of Mean Squared Error (MSE) for naval vessel propulsion system using whole data-set with training (70\%)-testing (30\%).

(threshold $=0.01$, stepmax $=100000$ ) provided by the R neural net library, the results did not converge. We tried to tune the net by increasing stepmax to 1000000 , and keeping the threshold the same (as increasing the threshold may affect accuracy). Still it did not converge. Increasing stepmax alone further could take a prohibitively large amount of time for our code to run without any guarantee of convergence. So, finally we tuned the neural net by increasing the threshold to 0.1 and stepmax to 1000000 and it converged. Even after compromising accuracy by increasing the threshold, the neural nets are able to optimize successfully. The simulation is performed with four different hidden layers architectures which are 8-6-4-2, 8-6-4, 8-6, and 6-4. Our results are shown in Table 1.

From the table it can be seen that with architecture 8-6-4-2 the Mean Square Error (MSE) for compressor decay state coefficient is 0.00017 and MSE for turbine decay state 


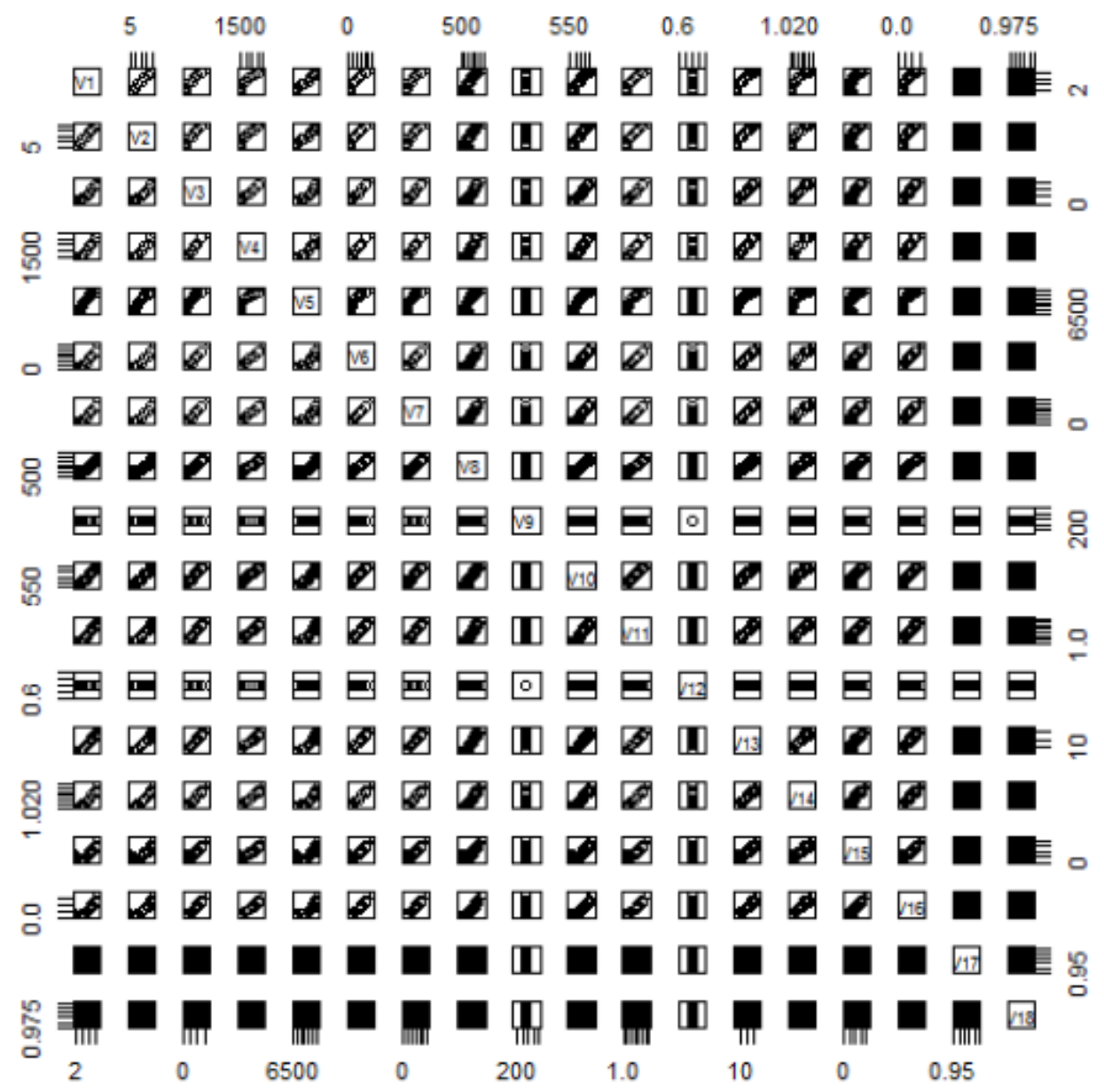

Fig. 4. Scatter plot matrix of input and output variables for visual representation.

coefficient is 0.00013 . The average MSE is 0.00015 . The table also shows that the architecture with 6-4 has failed to converge. The best MSE for prediction of the compressor decay state coefficient is obtained from architecture 8-6-4 (which is 0.00011) whereas the best MSE for the prediction of turbine decay state coefficient is from architecture 8-6-4-2 which is 0.00013 . The best result in terms of average MSE is from 8-6-4 and the MSE is 0.00014 and the neural network architecture is shown in Fig. 5.

The best performing neural network displayed in Fig. 5 shows a consistent decrease in the number of neurons in each layer of the neural network from the input to each of the hidden layers, followed by the result layer. This consistent decrease which starts with 16 inputs, followed by 86-4 neurons in the hidden layers, and 2 neurons in the output layer, shows an interesting architecture design, to which the successful results might be attributed. The first hidden layer is half the size of the input and the output layer is half the size of the last hidden layer. These results show that the best neural network architecture is the one that is decreasing in the hidden layer which is proportional to number of the inputs and to the number of outputs. The different architectures of the deep neural networks show some promising results which might be relevant to other neural networks for predictive maintenance systems or neural network architecture in general. 


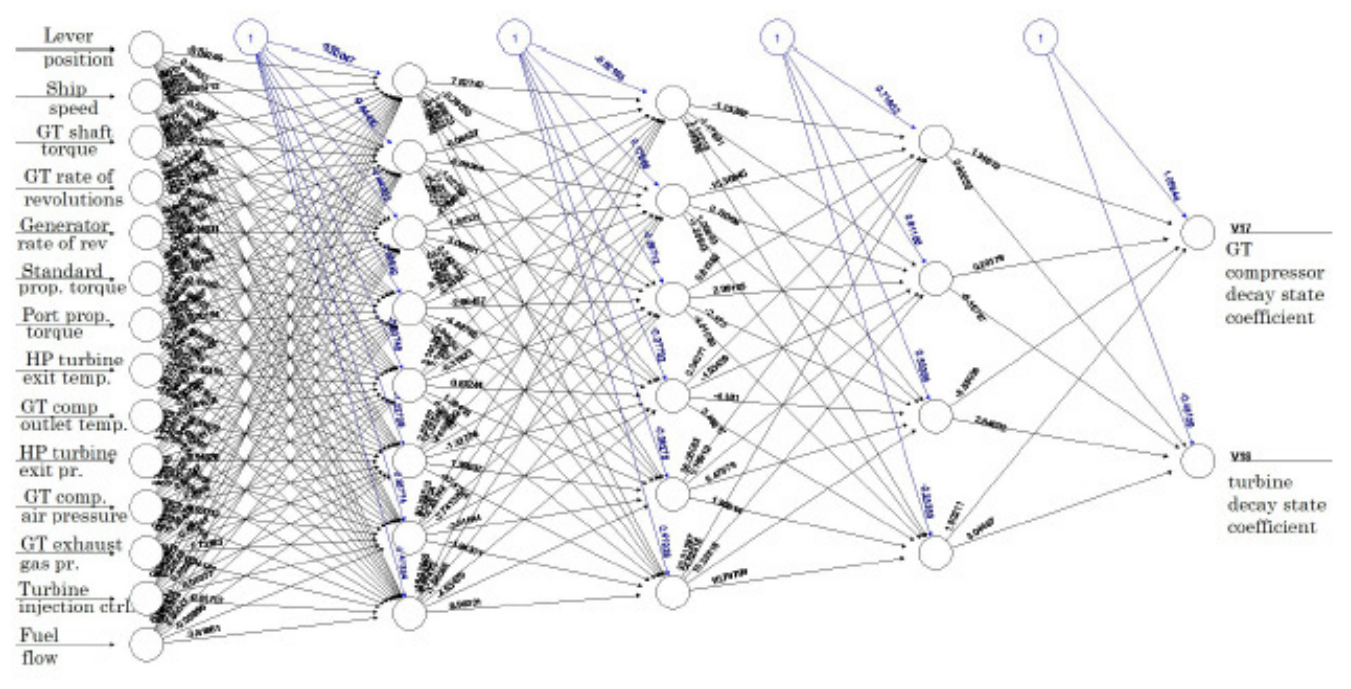

Fig. 5. The best network architecture in terms of average MSE.

Next, experiments were performed to analyze the dimensionality reduction by comparing ANN with the integration of Principle Component Analysis (PCA) [30] and ANN. PCA is a statistical method for orthogonal transformation which converts the data-set to linearly uncorrelated variables and it can identify redundant data. The motivation of integrating PCA into ANN is to eliminate correlated redundant data.

These experiments were performed with a reduced data-set (randomly selected data-set size 1,000 and 2,000). During the experiments with the whole data-set there is a compromise in terms of threshold. However, the reduced data-set is experimented with default values (threshold $=0.01$, stepmax $=100000$ ). Three different network architectures were used which are hidden layers with 5-4-3, 6-4-3, and 7-5-3. In addition, the performance of each architecture with each one of the output variables and with both variables was analyzed.

Table 2 and Table 3 show the comparison between ANN and PCA-ANN results for data-set size 1,000 and 2,000 respectively. In all cases, the performance of ANN is better than the performance of the integration of PCA and ANN. Moreover, from Table 3 it can be seen that the integrated PCA with ANN results for hidden layer with 5-4-3 with two outputs, 6-4-3 with second output and layer 7-5-3 for both output and second output failed to converge. This is an interesting observation which shows that reducing redundant data does not always help to achieve better results even though it is efficient in terms of computational complexity. The results show that it is more important to use a larger number of variables to represent a system than to use a smaller number of variables, even if the variable values are correlated. 


\begin{tabular}{|c|c|c|c|c|c|}
\hline \multirow{10}{*}{ 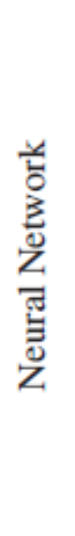 } & Architecture & Output & MSE1 & MSE2 & Average MSE \\
\hline & \multirow{3}{*}{$5-4-3$} & Both & 0.0003384 & 0.0004451 & 0.0003918 \\
\hline & & Output1 & 0.0001858 & & \\
\hline & & Output2 & & 0.0002898 & \\
\hline & \multirow{3}{*}{$6-4-3$} & Both & 0.0002123 & 0.0002229 & 0.0002176 \\
\hline & & Output1 & 0.0003042 & & \\
\hline & & Output2 & & 0.0003434 & \\
\hline & \multirow{3}{*}{$7-5-3$} & Both & 0.0001062 & 0.0002492 & 0.0001777 \\
\hline & & Output1 & 0.0002376 & & \\
\hline & & Output2 & & 0.0001386 & \\
\hline \multirow{9}{*}{  } & \multirow{3}{*}{$5-4-3$} & Both & 0.0052886 & 0.0129031 & 0.0090959 \\
\hline & & Output1 & 0.0011457 & & \\
\hline & & Output2 & & 0.0058067 & \\
\hline & \multirow{3}{*}{$6-4-3$} & Both & 0.0227793 & 0.0286877 & 0.0257335 \\
\hline & & Output1 & 0.0005859 & & \\
\hline & & Output2 & & 0.0110189 & \\
\hline & \multirow{3}{*}{$7-5-3$} & Both & 0.0020163 & 0.0153552 & 0.0086706 \\
\hline & & Output1 & 0.0007673 & & \\
\hline & & Output2 & & 0.0147235 & \\
\hline
\end{tabular}

Table 2. Comparison between different neural network architectures with PCA-cum-neural network hybrid architectures with reduced data-set size 1000 in terms of Mean Squared Error (MSE) for a naval vessel propulsion system

\section{CONCLUSION}

Maintenance demands a high level of expertise for a very complex system which is difficult enough to achieve for physical systems. Predictive maintenance is a domain where advanced computing techniques help to significantly increase system reliability. Maintenance strategies combined with statistical methods and automated learning algorithms may significantly improve overall performance of a system. A system like a propulsion plant of a naval destroyer always should be in proper working condition without any interruption, and at the same time its performance should be monitored continuously to avoid any unexpected downfall and to optimize performance.

In the present study, deep neural network and PCA integrated with deep neural network is used to predict the decay state coefficient of a GT compressor and GT turbine for naval vessel propulsion. The data for the experiment has been adapted from the UCI Machine Learning repository that contains 16 featured vectors (input) with two outputs. The backpropagation training algorithms are used for the present study. The simulation experiment is performed with four different deep neural networks (hidden layer such as 8-6-4-2, 8-6-4, 8-6 and 6-4). Our comparison with all the networks clearly depicts that the hidden layer structure with 8-6-4 network performs best in terms of average Mean Squared Error (MSE) to predict the decay state coefficient. In terms of compressor decay state coefficient, 8-6-4 is best, and for turbine decay state coefficient 8-6-4-2 is best. The network with 6-4 failed to converge in all cases. Thereafter, 
to understand the robustness of ANN, a reduced data set is used for the experiments with ANN and hybrid PCA-ANN. Results shows that ANN outperforms PCA-ANN.

\begin{tabular}{|c|c|c|c|c|c|}
\hline \multirow{10}{*}{ 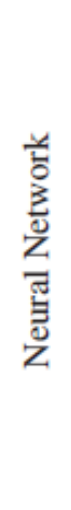 } & Architecture & Output & MSE1 & MSE2 & Average MSE \\
\hline & \multirow{3}{*}{$5-4-3$} & Both & 0.0000765 & 0.0002037 & 0.0001401 \\
\hline & & Output1 & 0.0000767 & & \\
\hline & & Output2 & & 0.0001400 & \\
\hline & \multirow{3}{*}{$6-4-3$} & Both & 0.0001001 & 0.0001055 & 0.0001028 \\
\hline & & Output1 & 0.0001307 & & \\
\hline & & Output2 & & 0.0001360 & \\
\hline & \multirow{3}{*}{$7-5-3$} & Both & 0.0001071 & 0.0001687 & 0.0001379 \\
\hline & & Output1 & 0.0000891 & & \\
\hline & & Output2 & & 0.0001249 & \\
\hline \multirow{8}{*}{  } & \multirow{3}{*}{$5-4-3$} & Both & & Convergence failed & \\
\hline & & Output1 & 0.0005811 & & \\
\hline & & Output2 & & 0.0134458 & \\
\hline & \multirow{3}{*}{$6-4-3$} & Both & 0.0036696 & 0.0157700 & 0.0097198 \\
\hline & & Output1 & 0.0006826 & & \\
\hline & & vatpats & & & \\
\hline & \multirow{2}{*}{$7-5-3$} & Both & & Convergence failed & \\
\hline & & Output2 & & Convergence failed & \\
\hline
\end{tabular}

Table 3. Comparison between different neural network architectures with PCA-cum-neural network hybrid architectures with reduced data-set size 2000 in terms of Mean Squared Error (MSE) for a naval vessel propulsion system

Though our experiment achieved good results, there is considerable scope for future research. Different learning algorithms can be used to train the network and results can be compared. Moreover, networks can be further tuned, adjusting the threshold to some lower value to get more accurate results. Experiment can also be performed to lower the time complexity to train the neural net model. While implementing the work in a real naval vessel, the concept of Internet of Things (IoT) can be used to gather streaming data from the system and system components in real time. The interval of time between two successive data collection events can be decreased, so that a massive amount of data becomes available as input. This massive amount of data then can be analyzed using big data analytics techniques and distributed computing, integrated with machine learning algorithms.

\section{REFERENCES}

[1] K. A. H. Kobbacy and D. P. Murthy, Complex System Maintenance Handbook. Springer Science \& Business Media, 2008.

[2] P. Compton and R. Jansen, "Knowledge in context: A strategy for expert system maintenance," in Australian Joint Conference on Artificial Intelligence. Springer, 1988, pp. 292-306.

[3] A. Widodo and B.-S. Yang, "Support vector machine in machine condition monitoring and fault diagnosis," Mechanical Systems and Signal Processing, vol. 21, no. 6, pp. 2560-2574, 2007. 
[4] G. A. Susto, A. Schirru, S. Pampuri, S. McLoone, and A. Beghi, "Machine learning for predictive maintenance: A multiple classifier approach," IEEE Transactions on Industrial Informatics, vol. 11, no. 3, pp.812-820, 2015.

[5] R. Barlow and L. Hunter, "Optimum preventive maintenance policies," Operations Research, vol. 8, no. 1,pp. 90-100, 1960.

[6] C. H. Lie and Y. H. Chun, "An algorithm for preventive maintenance policy," IEEE Transactions on Reliability, vol. 35, no. 1, pp. 71-75, 1986.

[7] T. J. McCoy, "Trends in ship electric propulsion," in Power Engineering Society Summer Meeting, 2002 IEEE, vol. 1. IEEE, 2002, pp. 343-346.

[8] K. Borisov, T. E. Calvert, J. A. Kleppe, E. Martin, and A. M. Trzynadlowski, "Experimental investigation of a naval propulsion drive model with the pwm-based attenuation of the acoustic and electromagnetic noise," IEEE Transactions on Industrial Electronics, vol. 53, no. 2, pp. 450-457, 2006.

[9] R. K. Hanson, "Applications of quantitative laser sensors to kinetics, propulsion and practical energy systems," Proceedings of the Combustion Institute, vol. 33, no. 1, pp. 1-40, 2011.

[10] U. Campora, M. Figari, M. Martelli, S. Vignolo, M. Viviani, and M. Ratto, "Propulsion plant simulation for fast military vessels," in Proceedings of HSMV 2011, 9th Symposium on High Speed Marine Vehicles, 2011.

[11] M. Martelli and M. Figari, "Real-time model-based design for CODLAG propulsion control strategies," Ocean Engineering, vol. 141, pp. 265-276, 2017.

[12] P. G. Hill and C. R. Peterson, Mechanics and Thermodynamics of Propulsion. Reading, MA, Addison Wesley Publishing Co., 1992, 764 p., 1992.

[13] D. W. Taylor, The Speed and Power of Ships: A Manual of Marine Propulsion. J. Wiley \& Sons, 1910, vol. 1.

[14] J. B. Black, "Marine propulsion system,” Jul. 17 1984, US Patent 4,459,873.

[15] V. Lamaris and D. Hountalas, "A general purpose diagnostic technique for marine diesel enginesapplication on the main propulsion and auxiliary diesel units of a marine vessel," Energy Conversion and Management, vol. 51, no. 4, pp. 740-753, 2010.

[16] G. Danek Jr, "State-of-the-art survey on hot corrosion in marine gas turbine engines," Naval Engineers Journal, vol. 77, no. 6, pp. 859-869, 1965.

[17] S. Hirdaris, Y. Cheng, P. Shallcross, J. Bonafoux, D. Carlson, B. Prince, and G. Sarris, "Considerations on the potential use of nuclear small modular reactor (SMR) technology for merchant marine propulsion,” Ocean Engineering, vol. 79, pp. 101-130, 2014.

[18] C. Schaffrin, "Energy-generating plant, particularly propeller-type ship's propulsion plant, including a solar generator," Aug. 10 1993, US Patent 5,235,266.

[19] C. Parsons, "The application of the compound steam turbine to the purpose of marine propulsion," Journal of the American Society for Naval Engineers, vol. 9, no. 2, pp. 374-384, 1897.

[20] J. F. Hansen, A. K. ${ }^{\circ}$ Adnanes, and T. I. Fossen, "Mathematical modelling of diesel-electric propulsion systems for marine vessels," Mathematical and Computer Modelling of Dynamical Systems, vol. 7, no. 3, pp. 323-355, 2001.

[21] R. A. Barr and R. Etter, "Selection of propulsion systems for high speed advanced marine vehicles," Tech. Rep., 1974.

[22] A. Lucifredi, C. Mazzieri, and M. Rossi, "Application of multiregressive linear models, dynamic kriging models and neural network models to predictive maintenance of hydroelectric power systems," Mechanical Systems and Signal Processing, vol. 3, no. 14, pp. 471-494, 2000.

[23] R. Javadpour and G. M. Knapp, "A fuzzy neural network approach to machine condition monitoring," Computers \& Industrial Engineering, vol. 45, no. 2, pp. 323-323, 2003.

[24] S.-j. Wu, N. Gebraeel, M. A. Lawley, and Y. Yih, "A Neural Network integrated decision support system for condition-based optimal predictive maintenance policy," IEEE Transactions on Syetems. Man, and Cybernetics Part A: Syetems and Humans, vol. 37, no. 2, 2007.

[25] J.-C. Han, S. Dutta, and S. Ekkad, Gas Turbine Heat Transfer and Cooling Technology. CRC Press, 2012.

[26] M. Altosole, "Simulation of the dynamic behaviour of a CODLAG propulsion plant," 2010.

[27] A. Coraddu, L. Oneto, A. Ghio, S. Savio, M. Figari, and D. Anguita, "Machine learning for wear forecasting of naval assets for condition-based maintenance applications," in Electrical Systems for Aircraft, Railway, Ship Propulsion and Road Vehicles (ESARS), 2015 International Conference on. IEEE, 2015, pp. 1-5. 
[28] A. Asuncion and D. Newman, "UCI machine learning repository," 2007.

[29] A. Coraddu, L. Oneto, A. Ghio, S. Savio, D. Anguita, and M. Figari, "Machine learning approaches for improving condition-based maintenance of naval propulsion plants," Journal of Engineering for the Maritime Environment, vol. 230, no. 1, pp. 136-153, 2014.

[30] V. Guralnik and W. K. Foslien, "Principal component analysis based fault classification," Nov. 4 2008, US Patent 7,447,609.

\section{Authors}

Palash Pal received B.Tech from University Institute of Technology, Burdwan University, West Bengal, India.

Rituparna Datta Dr. Rituparna Datta is working as a Computer Research Associate-II in the Department of computer Science, University of South Alabama, United States. Prior to that, he was a Operations Research Scientist in Boeing Research \& Technology (BR\&T), BOEING, Bangalore. He earned his Ph.D in Mechanical Engineering at Indian Institute of Technology (IIT) Kanpur in 2013.

Aviv Segev Aviv Segev is an Associate Professor in the Department of Computer Science of the School of Computing at the University of South Alabama. His research interest is looking for the DNA of knowledge, an underlying structure common to all knowledge, through analysis of knowledge models in natural sciences, knowledge processing in natural and artificial neural networks, and knowledge mapping between different knowledge domains. In 2004, he received a $\mathrm{Ph} . D$. from Tel-Aviv University in Technology and Information Systems.

Alec Yasinsac Alec Yasinsac is Professor and Dean of the School of Computing at the University of South Alabama. He joined the School of Computing in July 2008 after spending nine years on the faculty at Florida State University which followed a twenty year career in the United States Marines as a Data Systems and Communications Officer. He is also Co-founder of SAIT Laboratory and has operational experience in software development, information systems management, network engineering, and information security, having spent active duty tours in Japan, Korea, North Carolina, California, and Virginia. Alec a senior member of IEEE, a member of ACM, U.S. Public Policy Committee of the ACM, and the IEEE Computer Society. With research interests in electronic voting systems, Alec sat on the Florida Help America Vote Act Planning Committee and was on the Advisory Board for the 2007 National Academy of Engineering 2007 National Meeting Symposium On Electronic Voting. He led the first academic source code review in support of an election audit for the 2006 Florida United States Congressional District 13 race and led several other electronic voting security code reviews and systems security analysis for the Florida Department of State. He routinely contributes to national meetings and panels that address voting system security issues. 